\title{
Contralateral approach for clipping of bilateral anterior circulation aneurysms
}

\author{
Justin M. Caplan, M.D., Eric Sankey, B.S., David Gullotti, B.S., Joanna Wang, B.A., \\ Erick Westbroek, M.D., Brian Hwang, M.D., and Judy Huang, M.D. \\ Department of Neurosurgery, Johns Hopkins University School of Medicine, Baltimore, Maryland
}

Patients with bilateral anterior circulation aneurysms present a management challenge. These lesions may be treated in a staged manner or alternatively, for select patients, a contralateral approach may be utilized to treat bilateral aneurysms with a single surgery. In this narrated video illustration, we present the case of a 57-year-old woman with incidentally discovered bilateral aneurysms (left middle cerebral artery [MCA], left anterior choroidal artery and right MCA). A contralateral approach through a left pterional craniotomy was performed for microsurgical clipping of all three aneurysms. The techniques of pterional craniotomy, contralateral approach, microsurgical clipping and intraoperative angiography are reviewed.

The authors are grateful to Wuyang Yang, M.D. for his assistance.

The video can be found here: http://youtu.be/MIPlu3hQZkg.

KEYWORDS contralateral approach; microsurgical clipping; aneurysm; middle cerebral artery; anterior choroidal artery; lamina terminalis; video 